\title{
Editorial
}

\section{Special issue: Perspectives on intelligent agents research ... one year later}

\author{
SIMON PARSONS ${ }^{1}$ and ADELE HOWE \\ ${ }^{1}$ Department of Electronic Engineering, Queen Mary \& Westfield College, University of London, London E1 4NS, UK \\ ${ }^{2}$ Department of Computer Science, Colorado State University, Fort Collins, CO 80523, USA
}

\begin{abstract}
A year ago, we published a special issue of The Knowledge Engineering Review entitled "Perspectives on Recent Intelligent Agents Research as Viewed through Two Conferences". Our intention was to provide a snapshot of current research on intelligent agents - an area which continues to grow apace - by looking at the work presented at the 1997 instantiations of two of the major events in the Agent calendar. These were the International Conference on Autonomous Agents (Agents '97) and the International Conference and Exhibition on the Practical Application of Intelligent Agents and MultiAgent Technology (PAAM '97). The result was a group of six papers which together spanned the main areas covered by the conferences as well as identifying some of the challenges faced by the agents community as a whole. In particular, we had summaries by the organisers of both of the conferences, speculation on the future of robotics, surveys of expert assistants and electronic commerce, and a discussion of how agents could be used in workflow management.

The aim of this special issue is to extend that coverage and to take a further look at those challenges. We did this in much the same way as for the previous issue, by giving carte blanche to a group of authors we felt would have interesting, authoritative, and provocative things to say on the subject of agents research. As with the previous issue, the authors were invited based on their reputations and involvement in one of the 1998 versions of the two conferences: Agents ' 98 and PAAM '98. As before, this approach has given us a nicely diverse set of papers which neatly complement our previous selection. This complementarity is, in itself, interesting. For the previous special issue we made a point of selecting one conference which always takes place in the United States and one which always takes place in the United Kingdom. Our expectation was that we would expose some differences which might be attributed to the differences between the American viewpoint and the European viewpoint. No such continental differences emerged, though there was a wide range of opinion from author to author. This time, despite our attempts to interest authors from both sides of the Atlantic and including authors involved with each of the conferences, we have an entirely European authorship, with an equally diverse set of views.

These new papers fall into two broad classes - those which deal with specific issues in agents research illustrated with miniature surveys (in the sense that they cover less ground than typical papers for The Knowledge Engineering Review) and those which comment on the state of agent research as a whole.

In the first class, we have the papers by Conte (1999) and Rasmusson and Janson (1999). Taking Wooldridge's (1999) classification of the four sub-areas "that go under the 'agent' banner"-multiagent work which emerged from distributed AI and distributed problem solving, interface agents, mobile agents, and robotics - Conte comes primarily from the multi-agent corner, and her interest is in the social aspects of such systems. These are the aspects which allow agents to reason about, and hence be able to efficiently deal with, the other agents in their domain. As Conte argues, the ability to
\end{abstract}


interact with other agents is a very important aspect of agents which have to take account of others, whether those others are themselves artificial agents or are human. Indeed, the need to consider such aspects is one of the main things which distinguishes agent research from Good Old-Fashioned AI, in which it was widely assumed that systems had to have models of their environment, but in which such models rarely considered the impact of other systems ${ }^{1}$ on that environment, and certainly did not stoop to modelling those other systems explicitly. As a result, it seems to us that this work on taking account of other agents is one of the main contributions of agent research (two others are the work on agents' self-models and integrating disparate capabilities, e.g., sensing with action).

In considering these social issues, Conte's paper covers ground that was not considered in any depth by authors in the previous special issue. In fact, her paper could be considered as a detailed examination of one of the "social issue" themes mentioned by Crabtree (1998), in particular the second strand of his comment that:

It is vitally important that users trust their agents to work effectively on their behalf. The only way that this can be done is by building up a "relationship of trust" between the user and agent.

although she covers much wider issues as well. Rasmusson and Janson (1999) deal with a similar issue, but from a very different perspective. These authors are concerned with the use of agents in electronic commerce, and so can be seen as extending the coverage of the area provided by Guttman et al. (1998), and their main concern is the role of agents' self interest in providing help in electronic markets. The main thrust of their argument, at the risk of over-simplifying their careful analysis, is that self-interest is of vital importance in electronic markets in order to meet the best interests of those employing the agents, and that cooperation between agents is likely to be harmful to such ends. Thus, we must only build selfish agents if we are going to trust such agents to work for our best interests in electronic markets. ${ }^{2}$

In the class of papers which deal with the state of agent research in general, we have the papers by Luck (1999), Nwana and Ndumu (1999) and Wooldridge (1999). Interestingly, given the general enthusiasm for agent technologies, all three express a degree of pessimism.

Luck (1999) points out that most research into agents has fallen into two camps - it is either work on practical applications, which makes possibly over-simplifying assumptions, or it is work on complex logics, which have not been usefully applied. Nwana and Ndumu (1999) took us at our word in inviting controversy and write at length on the same point. While Luck concludes that although there has been little progress as yet there is an increasing amount of work which aims to bridge the divide, Nwana and Ndumu suggest that the outlook is considerably bleaker. For them, the community of agent researchers is simply ignoring a whole range of basic issues, such as developing suitable methodologies for building large agent systems and building usable ontologies, which are essential in the construction of the kind of practical agent systems that the field has been offering as its reason for existing. As a result, they feel that the field has yet to deal with the real issues, as they put it, "the devil in realising the promises of agent technology is in the details", and that these details have yet to be considered. More dire still, they conclude that instead of confronting these important details, researchers are largely either wasting their time fiddling with new and complicated logics which are irrelevant (falling into the trap that Russell labelled "premature mathematization"), or are busily re-inventing or re-labelling old ideas. The "rebranding" tendency also concerns Wooldridge (1999) who fears that, in the long term, the huge diversity of the number of systems being labelled as agents is going to lead to the computer science community at large concluding that the term "agent" is meaningless.

However, these pessimists are also believers in the long term viability of the field. Wooldridge points out that the flipside of the diversity is that it suggests that there is something very powerful in the concept of an agent, and that they definitely have a role if only as a useful abstraction in

\footnotetext{
${ }^{1}$ Other than nature if you happen to want to consider nature as a system.

${ }^{2}$ It is worth noting that rather different aspects of trust, which relate - not surprisingly considering the authors - quite closely with the points made by Conte, are covered in Falcone and Firozabadi (1999).
} 
modelling the world. Similarly, Nwana and Ndumu agree that the concept of agents is useful, and that the promised advantages can be delivered, albeit at the cost of tackling some rather hard, and possibly rather dull, problems. Luck, while agreeing with this last point, is confident that, even if all the benefits claimed for agent technology are never delivered, the search for them will necessitate a new set of tools and techniques which will benefit computer science as a whole.

\section{References}

Bekey, G, 1998. "On autonomous robots” The Knowledge Engineering Review 13(2) 143-146.

Conte, R, 1999. “Artificial social intelligence: a necessity for agent systems' developments” The Knowledge Engineering Review 14 (2).

Crabtree, B, 1998. "What chance software agents?” The Knowledge Engineering Review 13(2) 131-136.

Falcone, R and Firozabadi, BS, 1999. "The challenge of trust" The Knowledge Engineering Review 14(1).

Guttman, R, Moukas, AG and Maes, P, 1998. "Agent-mediated electronic commerce" The Knowledge Engineering Review 13(2) 147-159.

Johnson, WL and Hayes-Roth, B, 1998. "The first autonomous agents conference" The Knowledge Engineering Review 13(2) 137-142.

Luck, M, 1999. "From definition to deployment: what next for agent-based systems?" The Knowledge Engineering Review 14(2).

Nwana, HS and Ndumu, DT, 1999. "A perspective on software agents research" The Knowledge Engineering Review 14(2).

O'Brien, P and Wiegand, M, 1998. "Agent based process management: applying intelligent agents to workflow" The Knowledge Engineering Review 13(2) 161-174.

Rao, A, 1998. "A report on expert assistants at the Autonomous Agents conference" The Knowledge Engineering Review 13(2) 175-178.

Rasmusson, L and Janson, S, 1999. "Agents, self-interest, and electronic markets" The Knowledge Engineering Review 14(2).

Wooldridge, M, 1999. "Diversity and agent technology" The Knowledge Engineering Review 14(2). 